\title{
Nucleon-Nucleon Correlations and Final State Interaction in Inclusive Quasi-Elastic Electron Scattering off Nuclei at $x>1$
}

\author{
C. Ciofi degli $\operatorname{Atti}^{(a)}$ and S. Simula ${ }^{(b)}$ \\ ${ }^{(a)}$ Department of Physics, University of Perugia and \\ Istituto Nazionale di Fisica Nucleare, Sezione di Perugia, \\ Via A. Pascoli, I-06100 Perugia, Italy \\ ${ }^{(b)}$ Istituto Nazionale di Fisica Nucleare, Sezione Sanitá, \\ Viale Regina Elena 299, I-00161 Roma, Italy
}

\begin{abstract}
Inclusive quasi-elastic electron scattering off nuclei is investigated at high momentum transfer $\left(Q^{2}>1(\mathrm{GeV} / \mathrm{c})^{2}\right)$ and $x>1$ adopting a consistent treatment of nucleonnucleon correlations in initial and final states. It is shown that in case of light as well as complex nuclei the inclusive cross section at $1.3<x<2$ is dominated by the absorption of the virtual photon on a pair of correlated nucleons and by their elastic rescattering in the continuum, whereas at $x>2$ it is governed by the rescattering of the outgoing off-mass-shell nucleon in the complex optical potential generated by the ground state of the residual (A-1)-nucleon system.
\end{abstract}

PACS number(s) : 25.30.Fj

To appear in Phys. Lett. B. 
Inclusive quasi-elastic (QE) electron scattering off nuclei at high momentum transfer can provide non trivial information on the nuclear wave function. In particular, the kinematics region corresponding to $x>1+k_{F} / M \approx 1.3$ (where $k_{F}$ is the Fermi momentum and $x=Q^{2} / 2 M \nu$ the Bjorken scaling variable), is strongly affected by high momentum and high removal energy components of the nuclear wave function arising from nucleon-nucleon (NN) short-range and tensor correlations. Such a result has been obtained by several authors [1-5] using the plane wave Impulse Approximation (IA). Recently, however, it has been shown [6] that the Final State Interaction (FSI) of the struck nucleon, evaluated with an on-shell optical potential, totally overwhelms the effects from ground-state NN correlations. Nonetheless, in ref. [7] the relevance of NN correlations on the inclusive cross section in the region $1<x<2$ has been again advocated, arguing [2] that in such a region (where scattering from a nucleon at rest is kinematically forbidden) the contribution from a correlated NN pair in a nucleus should be proportional to the one in the deuteron (for which $x<2$ ). In order to further clarify the role played by NN correlations and FSI, we present here the first results of a calculation of the inclusive cross section based upon a novel approach for evaluating FSI relying on a consistent treatment of $\mathrm{NN}$ correlations in initial and final states.

The cross section for the inclusive process $A\left(e, e^{\prime}\right) X$ will be written hereafter in the following form

$$
\sigma^{(A)}=\frac{d^{2} \sigma}{d E_{e^{\prime}} d \Omega_{e^{\prime}}}=\sigma_{0}^{(A)}+\sigma_{1}^{(A)}
$$

where the contributions from different final nuclear states have been explicitly separated out, namely $\sigma_{0}^{(A)}$ describes the transition to the ground and one-hole states of the (A-1)-nucleon system and $\sigma_{1}^{(A)}$ the transition to more complex highly excited configurations. In what follows, the calculation of $\sigma_{0}^{(A)}$ and $\sigma_{1}^{(A)}$, within different levels of sophistication for the treatment of the final A-nucleon state, will be presented starting from the IA.

1. The Impulse Approximation. As is well known, the calculation of the inclusive cross section within the IA requires the knowledge of the nucleon spectral function $P(k, E)$, which represents the joint probability to find in a nucleus a nucleon with momentum $k \equiv|\vec{k}|$ and removal energy $E$. In presence of ground-state NN correlations $P(k, E)$ can be written as

$$
P(k, E)=P_{0}(k, E)+P_{1}(k, E)
$$

where the subindeces 0 and 1 have the same meaning as in eq. 1, i.e. $P_{0}$ includes the ground and one-hole states of the (A-1)-nucleon system and $P_{1}$ more complex configurations (mainly $1 \mathrm{p}-2 \mathrm{~h}$ states) which arise from $2 \mathrm{p}-2 \mathrm{~h}$ excitations generated in 
the target ground state by NN correlations. Thus, one can write [4]

$$
P_{0}(k, E)=\frac{1}{A} \sum_{\alpha \leq F} A_{\alpha} n_{\alpha}(k) \delta\left(E-\left|\epsilon_{\alpha}\right|\right)
$$

where $n_{\alpha}$ is the nucleon momentum distribution of the single particle state $\alpha$ having fractional occupation probability $\left(\int d \vec{k} n_{\alpha}(k)<1\right)$, energy $\epsilon_{\alpha}$ and number of nucleons $A_{\alpha}$. As for the correlated part $P_{1}$, we will consider in this paper high values of $k$ $\left(k>1.5 \mathrm{fm}^{-1}\right)$ and $E\left(E \sim k^{2} / 2 M\right)$, in which case, generalizing the results of ref. [8], we get

$$
\begin{aligned}
P_{1}(k, E)= & \sum_{N_{2}=n, p} \int d \vec{k}_{c m} n_{r e l}^{N_{1} N_{2}}\left(\vec{k}-\frac{\vec{k}_{c m}}{2}\right) n_{c m}^{N_{1} N_{2}}\left(\vec{k}_{c m}\right) \\
& \delta\left[E-E_{t h r}^{(2)}-\frac{A-2}{2 M(A-1)}\left(\vec{k}-\frac{A-1}{A-2} \vec{k}_{c m}\right)^{2}\right]
\end{aligned}
$$

where $n_{r e l}^{N_{1} N_{2}}\left(n_{c m}^{N_{1} N_{2}}\right)$ is the momentum distribution of the relative (center of mass) motion of the two nucleons in a correlated pair, and $E_{t h r}^{(2)}=M_{A-2}+2 M-M_{A}$ is the two-nucleon break-up threshold. Eq. 4 assumes that the (A-2)-nucleon system is in its ground state, which implies that the $\mathrm{cm}$ of the correlated pair moves in the mean field of the nucleus, so that only soft components of $n_{\mathrm{cm}}^{N_{1} N_{2}}$ contribute to $P_{1}$ (see for details refs. [8-9]). Eq. 4 quantitatively reproduces the high momentum and high removal energy parts of the many-body spectral function calculated for ${ }^{3} \mathrm{He}[1],{ }^{4} \mathrm{He}$ [10] and nuclear matter [6], as well as the momentum sum rule of complex nuclei [8-9]. Using eqs. 2-4 we write the IA cross section in the following forms, which will be useful for further elaboration

$$
\begin{aligned}
{\left[\sigma_{0}^{(A)}\right]_{I A} } & =\sum_{N=1}^{A} \int d \vec{k} d E \sigma_{e N} P_{0}^{N}(k, E) \delta\left[\nu+k^{0}-E_{\vec{k}+\vec{q}}\right] \\
& =\sum_{\alpha \leq F} \int_{0}^{\infty} d k k n_{\alpha}(k)\left(Z_{\alpha} \sigma_{e p}+N_{\alpha} \sigma_{e n}\right) \frac{\nu+k^{0}}{q}[\Delta(k)]_{I A} \\
{\left[\sigma_{1}^{(A)}\right]_{I A} } & =\sum_{N=1}^{A} \int d \vec{k} d E \sigma_{e N} P_{1}^{N}(k, E) \delta\left[\nu+k^{0}-E_{\vec{k}+\vec{q}}\right] \\
& =A \sigma_{M o t t} \sum_{N_{1} N_{2}=n, p} \int d \vec{k}_{c m} n_{c m}^{N_{1} N_{2}}\left(\vec{k}_{c m}\right) L^{\mu \nu}\left[W_{\mu \nu}^{N_{1} N_{2}}\right]_{I A}
\end{aligned}
$$

In eqs. $5-8 \nu(\vec{q})$ is the energy (three-momentum) transfer; $\vec{k}$ is the momentum of the nucleon in the lab system before interaction and $k^{0}=M_{A}-\sqrt{\left(M_{A}+E-M\right)^{2}+k^{2}}$ its off-shell energy; $E_{\vec{p}}=\sqrt{M^{2}+|\vec{p}|^{2}} ; \sigma_{e N}$ is the electron - (off-shell) nucleon cross 
section; $[\Delta(k)]_{I A}$ is

$$
\begin{aligned}
{[\Delta(k)]_{I A} } & =\int_{E_{p}^{-}}^{E_{p}^{+}} d E_{p} \frac{E_{p}}{\nu+k_{\alpha}^{0}} \delta\left(\nu+k_{\alpha}^{0}-E_{p}\right) \\
& =\Theta\left(E_{p}^{+}-\nu-k_{\alpha}^{0}\right) \Theta\left(\nu+k_{\alpha}^{0}-E_{p}^{-}\right)
\end{aligned}
$$

where $E_{p}^{ \pm}=\sqrt{M^{2}+(k \pm q)^{2}}$. In eq. $8 L_{\mu \nu}$ is the (reduced) leptonic tensor and $\left[W_{\mu \nu}^{N_{1} N_{2}}\right]_{I A}$ the hadronic tensor of a correlated pair, which can be written as

$$
\begin{aligned}
{\left[W_{\mu \nu}^{N_{1} N_{2}}\right]_{I A}=} & \sum_{f_{12}^{0}} \sum_{\beta_{12}}\left[\left\langle\beta_{12}\left|j_{\mu}^{N_{1}}+j_{\mu}^{N_{2}}\right| f_{12}^{0}\right\rangle\right]^{*} \sum_{\beta_{12}^{\prime}}\left[\left\langle\beta_{12}^{\prime}\left|j_{\nu}^{N_{1}}+j_{\nu}^{N_{2}}\right| f_{12}^{0}\right\rangle\right] \\
& \delta\left[\nu+k_{c m}^{0}-\sqrt{\left(M_{2}^{f_{12}^{0}}\right)^{2}+\left(\vec{k}_{c m}+\vec{q}\right)^{2}}\right]
\end{aligned}
$$

where $j_{\mu}^{N}$ is the nucleon current, $k_{c m}^{0}=M_{A}-\sqrt{M_{A-2}^{2}+\left|\vec{k}_{c m}\right|^{2}},\left|\beta_{12}\right\rangle$ is the relative wave function of a correlated pair and $\left|f_{12}^{0}\right\rangle$ its plane wave final state. It should be pointed out that, whereas eqs. 5 and 7 are valid for any spectral function, eqs. 6 and 8 results from eqs. 3 and 4 , respectively. In particular, eq. 8, whose usefulness will be clear later on, is based upon the assumption that final and initial A-nucleon states factorize as follows: $\left|\Psi_{A}^{f}\right\rangle \sim \hat{A}\left[\left|f_{12}^{0}\right\rangle\left|\vec{P}_{c m}\right\rangle\left|\Psi_{A-2}^{f}\right\rangle\right]$ and $\left|\Psi_{A}^{0}\right\rangle \sim \hat{A}\left[\left|\beta_{12}\right\rangle\left|\chi_{12}^{c m}\right\rangle\left|\Psi_{A-2}^{0}\right\rangle\right]$ where $\hat{A}$ is a proper antisymmetrization operator, $\left|\chi_{12}^{c m}\right\rangle$ the $\mathrm{cm}$ wave function of a correlated pair and $\left|\vec{P}_{c m}\right\rangle$ its plane wave final state (note that the factorized form for $\left|\Psi_{A}^{0}\right\rangle$ is the basic ingredient for obtaining eq. $4[8]$ and that, using eq. 10 in eq. 8, eq. 7 is recovered in terms of the nucleon spectral function $P_{1}$ given by eq. 4 with $\left.n_{r e l}^{N_{1} N_{2}}\left(\vec{k}_{r e l}\right)=\sum_{\beta_{12}}\left|\left\langle\beta_{12} \mid \vec{k}_{r e l}\right\rangle\right|^{2}\right)$. The IA processes corresponding to eqs. 5 and 7 are depicted in fig. 1a and 1b, respectively, and the results of the calculations for ${ }^{2} \mathrm{H},{ }^{4} \mathrm{He}$ and ${ }^{56} \mathrm{Fe}$ at $Q^{2} \sim 2(\mathrm{GeV} / \mathrm{c})^{2}$ are shown by the dotted lines in fig. 2. It can be seen that, in agreement with previous calculations [1-6], the IA sizably underestimates the cross section at $x>1.3$; this is a very well known fact (common to both few-body systems [1], complex nuclei [4-5] and nuclear matter [6]) which is ascribed to the lack of any FSI within the IA (note that, unlike the region at $x \leq 1$, the effects from meson exchange currents are strongly suppressed at high momentum transfer and $x>1$ ). In what follows the FSI will be evaluated by a novel approach based upon the observation that the FSI involving two-nucleons emitted because of ground-state NN correlations (process of fig. 1c, called two-nucleon rescattering) should be different from the FSI involving the nucleon knocked-out from shell model states (process of fig. 1d, called single nucleon rescattering ).

2. Two-nucleon rescattering. The basic assumption underlying eq. 4 is that two nucleons are locally correlated at short separations, with their $\mathrm{cm}$ being apart from the spectator (A-2)-nucleon system $[2,8]$. The two-nucleon correlation in a nucleus reminds the one acting in the deuteron; indeed, in agreement with the results of 
many-body calculations [11], the nucleon momentum distribution of complex nuclei at $k>1.5 \mathrm{fm}^{-1}$, as predicted by eq. 4 , turns out to be the properly rescaled deuteron momentum distribution [9]. Therefore, at high values of $k$ and $E$ the absorption of the virtual photon by a correlated pair, which at $x>1.3$ is the dominant mechanism in the IA, is expected to resemble the one in the deuteron; if so, such a deuteron-like picture of the initial state should be extended also to the final state by allowing the two nucleons to elastically rescatter as depicted in fig. 1c. An important difference with respect to the case of the deuteron is that a correlated pair in a nucleus is bound and moves in the field created by the other nucleons. The details of the calculation of the two-nucleon rescattering in the medium will be presented elsewhere [12]; here, it suffices to say that the basic step of our approach is the replacement of the IA hadronic tensor $\left[W_{\mu \nu}^{N_{1} N_{2}}\right]_{I A}$ by the interacting one $W_{\mu \nu}^{N_{1} N_{2}}$, which is nothing but eq. 10 with the plane wave state $\left|f_{12}^{0}\right\rangle$ replaced by the exact NN scattering wave function $\left|f_{12}\right\rangle$ (note that the two-nucleon rescattering process cannot be expressed in terms of a spectral function). It can be seen from eq. 10 that medium effects on the interacting hadronic tensor are generated by the energy conserving $\delta$ function, in that the intrinsic energy available to the pair is fixed by its cm four-momentum, and, therefore, by the momentum distribution $n_{c m}^{N_{1} N_{2}}$ appearing in eq. 8; even if the cm motion is neglected $\left(n_{c m}^{N_{1} N_{2}}=\delta\left(\vec{k}_{c m}\right)\right)$ [2], medium effects still would remain through the quantity $k_{c m}^{0}$. We have calculated the inclusive cross section for the deuteron using the RSC NN potential [13], taking into account the rescattering in S, P and D partial waves; then, using the same two-nucleon amplitudes $\left\langle\beta_{12}\left|j_{\mu}^{N_{1}}+j_{\mu}^{N_{2}}\right| f_{12}\right\rangle$, we have computed the cross section $\sigma_{1}^{(A)}$ for complex nuclei. The results are shown by the dashed lines in fig. 2: it can be seen that at $1.3<x<2$ the process of two-nucleon rescattering brings theoretical predictions in good agreement with experimental data. The most striking aspect of our results is that the same mechanism which explains the deuteron data, does the same in a complex nucleus, provided the A dependence due to $n_{c m}^{N_{1} N_{2}}$ and $k_{c m}^{0}$ (clearly exhibited in fig. 2) is properly considered. It should be pointed out that our results hold for the whole set of kinematics considered in refs. [14-15].

3. Single nucleon rescattering. It can be seen from fig. 2 that the two-nucleon rescattering is not able to describe the experimental data at $x>2$. This fact is not surprising, because at $x>2$ more than two nucleons should be involved in the scattering process. We have mocked up this process by considering the motion of the nucleon, knocked-out from shell model states, in the optical potential generated by the ground state of the (A-1)-nucleon system (fig. 1d). Such an approach amounts to replace the quantity $[\Delta(k)]_{I A}$ in eq. 6 by the quantity

$$
[\Delta(k)]_{o p t}=-\int_{E_{p}^{-}}^{E_{p}^{+}} d E_{p} \frac{E_{p}}{\pi\left(\nu+k_{\alpha}^{0}\right)} \frac{\operatorname{Im} V_{o p t}}{\left[\nu+k_{\alpha}^{0}-E_{p}-R e V_{o p t}\right]^{2}+\left[\operatorname{Im} V_{o p t}\right]^{2}}
$$

resulting from the eikonal approximation for the nucleon propagator [16] (note that the two-nucleon rescattering (diagram 1c) is not included in the process described by diagram $1 \mathrm{~d}$, because the two-nucleon rescattering is not a multiple scattering process, i.e., it does not contribute to an optical potential). The quantity $[\Delta(k)]_{\text {opt }}$ (eq. 11), 
calculated with the common on-shell choice $V_{\text {opt }}=-\rho v_{N} \sigma_{N N}\left(i+\alpha_{N N}\right) / 2(\rho$ is the nuclear density, $v_{N}$ the nucleon velocity, $\sigma_{N N}$ the total NN cross section and $\alpha_{N N}$ the ratio of the real to the imaginary part of the forward NN scattering amplitude) is compared in fig. 3a with $[\Delta(k)]_{I A}$ (eq. 9): it can be seen that, whereas the latter vanishes for $k<k_{\min }\left(k_{\min }>k_{F}\right.$ at $\left.x>1.3\right),[\Delta(k)]_{\text {opt }} \neq 0$ even when $k<k_{\min }$, so that the low momentum part of the nucleon momentum distribution contributes to the cross section, resulting in a sizable increase of the low-energy tail of the QE peak in sharp disagreement with experimental data $[17,6]$. However, treating FSI at $x>1$ in terms of on-shell optical potentials is not justified $[2,7,17]$. Indeed, the struck nucleon, having four-momentum squared $p^{\prime 2} \cong(\nu+M-E)^{2}-(\vec{k}+\vec{q})^{2}$, can be either on-mass-shell $\left(p^{\prime 2}=M^{2}\right)$ or off-mass-shell $\left(p^{\prime 2} \neq M^{2}\right)$ depending on the values of $k$ and $E$. Initial configurations with $k<k_{\min }$ always give rise to intermediate off-shell (virtual) nucleons, whose rescattering amplitudes are expected to decrease with virtuality, for off-shell nucleons have to interact within short times. In order to take into account off-shell effects, we have included in $V_{\text {opt }}$ a suppression factor of the type

$$
V_{\text {opt }}=-\frac{1}{2} \rho v_{N} \sigma_{N N}\left(i+\alpha_{N N}\right) e^{-\delta\left|M^{2}-p^{2}\right|}
$$

where the parameter $\delta$ is the same for all kinematics considered in this paper. The details will be given elsewhere [12]; here, we would like to stress that, using the suppression factor in eq. 12, i) the calculated longitudinal response of nuclear matter agrees with the "exact" one obtained in ref. [18] within the orthogonalized correlated basis method, and ii) the asymptotic limit $\left(Q^{2} \rightarrow \infty\right)$ of eqs. 9 and 11 coincide, as theoretically argued [19]. The quantity $[\Delta(k)]_{\text {opt }}$ (eq. 11) calculated using eq. 12, is shown in fig. 3a and it can be seen that the off-shell corrections strongly suppress the contributions from low-momentum nucleons. The effects of the various choices of $\Delta(k)$ on the cross section $\sigma_{0}^{(A)}$ are shown in fig. 3b, which clearly exhibits the damping generated by off-shell corrections. To sum up, at $x>1$ the struck nucleon propagates in the medium with high virtuality, so that any calculation of its rescattering in terms of on-shell optical potentials has little to recommend itself. Our results, including all diagrams 1a-1d, are presented in fig. 4, where the nuclear scaling function $F(y, q)$ (see ref. [1]) is plotted against $q^{2}$ for a fixed value of the scaling variable $y$ (we remind the reader that $y=0(<0)$ corresponds to $x=1(>1))$. The agreement with the experimental data is good and holds in the whole low-energy side of the QE peak.

In conclusion, unlike previous approaches based upon single nucleon rescattering in the final state $[6,17]$, we have also considered the two-nucleon rescattering. The main results of our calculations are as follows: i) at $x>2$ the inclusive cross section is dominated by $\gamma^{*}$ absorption by a low momentum nucleon and by the rescattering of the highly virtual produced nucleon in the complex optical potential generated by the ground- state of the residual (A-1)-nucleon system; ii) at $1.3<x<2$ the cross section is strongly affected by NN correlations, being governed by $\gamma^{*}$ absorption by a pair of correlated nucleons and by their rescattering in the continuum; both initial state correlations and final state interaction resemble the ones occurring in the deuteron, apart from the cm motion and the binding of the pair in a complex nucleus. 
We are grateful to L. Frankfurt, B.Z. Kopeliovich, T.-S. H. Lee, M. Strikman and D. Treleani for many enlightening discussions. One of us (C. C.d.A.) would like to acknowledge G. West for his kind hospitality at LANL, where part of the manuscript was completed.

\section{References}

[1] C. Ciofi degli Atti, E. Pace and G. Salmé: Phys. Lett. 127B (1983) 303; Phys. Rev. C43 (1991) 1155.

[2] L.L. Frankfurt and M.I. Strikman: Phys. Rep. 160 (1988) 235.

[3] X. Ji and R. McKeown: Phys. Lett. 236B (1990) 130.

[4] C. Ciofi degli Atti, S. Liuti and S. Simula: Phys. Rev. C41 (1990) R2474.

[5] C. Ciofi degli Atti, D.B. Day and S. Liuti: Phys. Rev. C46 (1992) 1045.

[6] O. Benhar, A. Fabrocini, S. Fantoni, G.A. Miller, V.R. Pandharipande and I. Sick: Phys. Rev. C44 (1991) 2328.

[7] L Frankfurt, M. Strikman, D.B. Day and M. Sargsyan: Phys. Rev. C48 (1993) 2451.

[8] C. Ciofi degli Atti, S. Simula, L. Frankfurt and M. Strikman: Phys. Rev. C44 (1991) R7.

[9] M. Borromeo, C. Ciofi degli Atti and S. Simula: to be published.

[10] H. Morita and T. Suzuki: Prog. Theor. Phys. 86 (1991) 671.

[11] S.C. Pieper, R.B. Wiringa and V.R. Pandharipande: Phys. Rev. C46 (1992) 1741.

[12] C. Ciofi degli Atti and S. Simula: to be published.

[13] R.V. Reid: Ann. Phys. (N.Y.) 50 (1968) 411.

[14] W. Schutz et al.: Phys. Rev. Lett. 38 (1977) 259; S. Rock et al.: ibid. 49 (1982) 1139; S. Rock : private communication.

[15] D.B. Day et al.: Phys. Rev. Lett. 59 (1987) 427; Phys. Rev. C40 (1989) 1011.

[16] S.A. Gurvitz and A.S. Rinat: Phys. Rev. C35 (1987) 696.

[17] T. Uchiyama, A.E.L. Dieperink and O. Scholten: Phys. Lett. 233B (1989) 31.

[18] A. Fabrocini and S. Fantoni: Nucl. Phys. A503 (1989) 375.

[19] S.A. Gurvitz, A.S. Rinat and R. Rosenfelder: Phys. Rev. C40 (1989) 1363; E. Pace, G. Salmé and G.B. West: Phys. Lett. 273B (1991) 205.

[20] S. Galster et al.: Nucl. Phys. B32 (1971) 221.

[21] T. De Forest: Nucl. Phys. A392 (1983) 232. 


\section{Figure Captions}

Fig. 1. Processes contributing to the $A\left(e, e^{\prime}\right) X$ cross section: a) one-nucleon emission within the IA; b) virtual photon absorption by a correlated NN pair within the IA; c) elastic two-nucleon rescattering between the emitted nucleons of a correlated pair; d) single nucleon rescattering of a nucleon knocked-out from shell model states.

Fig. 2. Inclusive cross sections at $Q^{2} \sim 2(\mathrm{GeV} / \mathrm{c})^{2}[14-15]$ versus the energy transfer $\nu$. Calculations have been performed using the free nucleon form factors of ref. [20], the cc1 prescription of ref. [21] for $\sigma_{e N}$ and the RSC potential [13] for the NN interaction. Dotted line: IA (figs. $1 \mathrm{a}+1 \mathrm{~b}$ ); dashed line: IA + two-nucleon rescattering (figs. 1a-1c); dot-dashed line: contribution from nucleon inelastic channels estimated as in ref. [5].

Fig. 3. (a) The quantity $\Delta(k)$ for the process ${ }^{56} F e\left(e, e^{\prime}\right) X$ plotted versus the nucleon momentum $k$ at $x=1.5$. Dotted line: eq. 9 (fig. 1a); dashed line: eq. 11 (figs. $1 \mathrm{a}+1 \mathrm{~d})$ calculated using $V_{\text {opt }}=-\rho v_{N} \sigma_{N N}\left(i+\alpha_{N N}\right) / 2$ (see text); solid line: eq. 11 (figs. $1 \mathrm{a}+1 \mathrm{~d}$ ) calculated using the off-shell optical potential given by eq. 12. (b) The cross section corresponding to the three different calculations of $\Delta(k)$ shown in fig. 3a. In both figures $Q^{2} \sim 2(\mathrm{GeV} / \mathrm{c})^{2}$.

Fig. 4. Nuclear scaling function $F(y, q)$ for ${ }^{2} \mathrm{H}$ (a), ${ }^{4} \mathrm{He}$ (b) and ${ }^{56} \mathrm{Fe}$ (c) versus the squared three-momentum transfer $q^{2}$ for a fixed value of the scaling variable $y=-0.4 \mathrm{GeV} / \mathrm{c}$ [1]. Dotted line: IA (figs. $1 \mathrm{a}+1 \mathrm{~b}$ ); dashed line: correlated NN pair contribution (figs. $1 \mathrm{~b}+1 \mathrm{c}$ ); dot-dashed line: mean field contribution (figs. $1 \mathrm{a}+1 \mathrm{~d}$ ); solid line: IA + full final state interaction (figs. 1a - 1d). The experimental points denoted by a full dot correspond to the kinematics of fig. 2 and, particularly, to $\nu=$ 0.86, 0.82, $0.78 \mathrm{GeV}$ for ${ }^{2} \mathrm{H},{ }^{4} \mathrm{He}$ and ${ }^{56} \mathrm{Fe}$, respectively. 
This figure "fig1-1.png" is available in "png" format from: http://arxiv.org/ps/nucl-th/9403001v1 
This figure "fig2-1.png" is available in "png" format from: http://arxiv.org/ps/nucl-th/9403001v1 
This figure "fig1-2.png" is available in "png" format from: http://arxiv.org/ps/nucl-th/9403001v1 
This figure "fig2-2.png" is available in "png" format from: http://arxiv.org/ps/nucl-th/9403001v1 